\title{
Epidemiology, Demographics, Triptan Contraindications, and Prescription Patterns of Patients with Migraine: A German Claims Database Study
}

Astrid Gendolla $\cdot$ Nadine Rauer · Susanne Kraemer - Inka Schwerdtner •

Andreas Straube

Received: August 8, 2021 / Accepted: November 17, 2021 / Published online: November 27, 2021

(C) The Author(s) 2021

\section{ABSTRACT}

Introduction: Migraine is a neurological disease with a considerable economic and societal burden that negatively impacts quality of life and productivity. Triptans are potent serotonin receptor agonists widely used to treat migraine attacks. Little is known about German patients with migraine diagnosed with triptan contraindications or those who discontinue triptans.

Methods: This retrospective observational study identifies adults with migraine from a German sickness fund database (2010-2018). Migraine prevalence was calculated for the database population and extrapolated to the German Statutory Health Insurance (SHI) population. Medication use, proportion and demographics of patients with triptan contraindications, prevalence of triptan discontinuation and use of triptans by patients with contraindications were analysed.

\section{A. Gendolla}

Private Practice, Essen, Germany

N. Rauer · S. Kraemer · I. Schwerdtner Lilly Deutschland GmbH, Bad Homburg, Germany

A. Straube $(\square)$

Department of Neurology, Klinikum Grosshadern,University Hospital, Ludwig-

Maximilian-University, 81377 Munich, Germany

e-mail: andreas.straube@med.uni-muenchen.de
Results: In total 120,170 patients with migraine were identified in the database population, of whom $77.7 \%$ were female; migraine prevalence was $2.0 \%$ (male) and $7.3 \%$ (female), leading to an extrapolated 2,923,979 patients with migraine in the entire German SHI population (2019); of these, $14.5 \%$ had $\geq 1$ triptan contraindication. The most frequent contraindication was a history of stroke/transient ischaemic attack. However, an extrapolated $25.9 \%$ of patients who had a triptan contraindication received a triptan prescription following diagnosis. We calculated that 975,698 patients in the entire German SHI population had ever received a triptan, of whom 596,364 did not receive a triptan prescription in the follow-up year. Of these 596,364 individuals, $96.6 \%$ continued to receive a migraine diagnosis after their last triptan prescription. These 'triptan discontinuers' were predominantly female (82.6\%). Most patients utilized only one specific triptan, with a large majority of ongoing triptan users who had used this specific triptan receiving $>4$ prescriptions.

Conclusion: We confirm the existence of German patients with migraine and unmet therapeutic needs. These are patients diagnosed with triptan contraindications or patients who have discontinued triptan use despite continued migraine attacks. More research is needed to ascertain reasons for triptan discontinuation and the risk of triptan use by patients with contraindications. 
Keywords: Claims study; Contraindications; Epidemiology; German population; Migraine; Triptans

\section{Key Summary Points}

\section{Why carry out this study?}

Migraine is a neurological disease with a high disease burden and is a major publichealth concern.

Triptans are a class of serotonin receptor (5-HT 1B/1D) agonists commonly used as an acute treatment during an individual migraine attack to relieve pain and disability and to reduce the duration of the attack.

Very little is known about the proportion of German patients who may require alternative therapeutic options for the treatment of migraine attacks due to issues with existing contraindications and insufficient efficacy and tolerability related to the use of triptans.

The aim of this study was to use administrative claims data to describe the proportion of German patients with migraine diagnosed with triptan contraindications and the proportion of patients with contraindications who continue to use triptans, and to characterize the patient population discontinuing triptan use despite subsequent migraine diagnoses.

\section{What was learned from the study?}

Our analysis confirmed the existence and provided a description of a population of German patients with migraine who discontinue triptans despite subsequent migraine diagnoses.

We report the prevalence of triptan contraindications and the concomitant use of triptans by individuals with these contraindications in the German migraine population.

\section{INTRODUCTION}

Migraine is a primary headache disorder characterized by unilateral or bilateral, moderate-tosevere headaches with additional symptoms which can include aura, photophobia, phonophobia, osmophobia, nausea or vomiting $[1,2]$. Migraine is ranked among the top ten most prevalent diseases in over 195 countries and territories, with an estimated 1.33 billion individuals affected worldwide and a global prevalence of $11.6 \%$ [3, 4]. Headache disorders are a major public health concern typically affecting adults during their most productive years of life. In 2019, headache disorders were the cause of $5.4 \%$ total years lived with disability (YLDs) worldwide, of which migraine accounted for $88.2 \%$ [5]. Migraine is ranked as the second most common cause of disability (all sexes, all ages) as measured by YLDs, and it continues to be ranked as the leading cause of disability in women aged 15-49 years [5-7].

Migraine treatment strategies include acute medications aimed at reducing the symptoms of a migraine attack and prophylactic therapy (pharmacological and non-pharmacological) designed to reduce attack frequency and severity. Acute medication consists of analgesics, non-steroidal anti-inflammatory drugs (NSAIDs), antiemetics and triptans, all of which should be administered at migraine onset. Triptans are a broad class of potent serotonin receptor (5-HT 1B/1D) agonists administered early during a migraine attack and are considered first-line therapy for individuals who do not respond sufficiently to acute medications such as NSAIDs, analgesics, antiemetics or others. First approved in Europe in 1991, there are currently seven different triptans available for the treatment of acute migraine in Germany [8].

New acute migraine medications with novel mechanisms of action are becoming available and should be particularly helpful for the treatment of those patients with either triptan contraindications and/or who experience insufficient efficacy or tolerability with triptans [9]. However, there is no clear statement in the literature on the number or demographics of German patients with migraine who display an 
insufficient response to triptans or who have contraindications that would limit their use of triptans. Administrative/claims data are collected primarily for billing and reimbursement purposes, and the analysis of these data is one approach to identify and study the diagnosis and prescriptions patterns associated with a specific patient population. Analyses based on claims data are sources of information that provide insights into current, real-life patient care practices and can assist in the decisionmaking process for healthcare stakeholders, researchers and policy-makers [10].

The aims of this administrative claims-based study were: (1) to describe the prevalence and demographic characteristics of patients with migraine in Germany, (2) to uncover the proportion of patients with migraine diagnosed with triptans indications, (3) to describe the population of patients with migraine who continue to use triptans despite the existence of contraindications, (4) to outline the population of patients with migraine that discontinue prescribed triptan use and (5) to gain an understanding of triptan and overall medication prescription patterns of patients with migraine who have interacted with the German healthcare system.

\section{METHODS}

\section{Study Design and Data Source}

This was a retrospective, observational study based on claims data from a statutory German sickness fund database which was drawn from seven national company health insurance funds with a total of 5.6 million insured people ("Betriebskrankenkassen" [BKK]). Data for our analyses were drawn from administrative claims information entered by the treating physician and compiled by the BKK. This information contained longitudinal data of insured individuals with respect to all areas of services refunded by the German Statutory Health Insurance (SHI) scheme, including data on in- and out-patient care, prescriptions and sick leave. Information on drug prescriptions and hospitalizations was documented daily, and diagnoses made by the healthcare professional group were provided on a quarterly basis. The database allowed for the analysis of groups of patients with defined characteristics (e.g. those with a specific disease or prescriptions of specific medications, or combinations of characteristics) at a specific date (index) and comparisons between groups. This database was representative (age and sex) of the total German population insured by the SHI scheme "Gesetzliche Krankenversicherung"). The overall study period lasted from January 2010 to December 2018. The migraine prevalence for the database population was calculated for the year 2017 (index). The years 2010-2017 were used for the analysis of triptan prescriptions and the presence of triptan contraindications. The year 2018 was used as a follow-up period in the analysis of triptan use/discontinuation patterns. Access to these regulated data was requested and obtained from the BKK, which had no further involvement in the analyses. Anonymized data from the electronic databases of the collaborating SHI funds were collected in accordance with an approved data privacy concept. The use of the fully anonymized secondary data for health services research was compliant with German federal law; therefore, institutional review board/ethical approval was not required $[40,41]$.

\section{Cohort Selection}

Patients who met the following criteria were included in our analysis: (1) continuously insured between 2010 and 2018; (2) $\geq 18$ years of age in 2017; and (3) having an assured migraine diagnosis in 2017 (International Classification of Disease 10th Edition; German Modification [ICD-10-GM] code G43) in in-patient (principal and secondary diagnoses) and/ or out-patient care sectors, or in the sick leave data. For out-patient and in-patient secondary diagnoses and sick leave diagnoses, a migraine diagnosis was considered only if there was an additional migraine diagnosis during the study period, within 1 year, but in a different quarter (M2Q criterion: "Mindestens zwei Quartale", meaning 'at least 2 quarters'). For outpatient 
data, only diagnoses marked by "G" ("gesichert", meaning 'assured') were included. For in-patient data, we considered all primary and secondary diagnoses; for sick leave data, we considered the single primary diagnosis [11]. From a total of 5,627,527 insured patients identified in the database, we focused our analysis on those 120,170 patients who met the inclusion criteria described above (Fig. 1a). These 120,170 adult patients with migraine were evaluated for the presence of contraindications. The list of contraindications analysed in the study was adapted from the summary of product characteristics (SmPC) of all triptans available in Germany. In contrast to the procedure for identifying patients with migraine, the $\mathrm{M} 2 \mathrm{Q}$ criterion was not applied to contraindications representing an acute event (e.g. heart attack). To study patients with migraine who discontinued their triptan use, we first identified patients with migraine without triptan contraindications and those with $\geq 1$ triptan prescription prior to 2018 (2010-2017). To further understand the population who discontinued triptan, we focused on those patients who received a migraine diagnosis following triptan discontinuation. The year 2018 served as a follow-up period for patients with migraine with triptan prescriptions in 2017 to estimate the possible non-refilling of their triptan prescriptions. This approach ensured that each patient was monitored for at least 1 year following their last triptan prescription. Additionally, the prescription data of all acute and preventive medications was reported for the year 2017. a

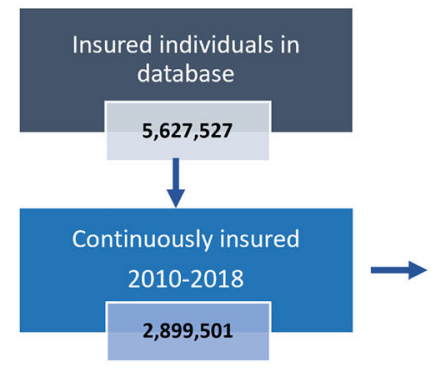

C

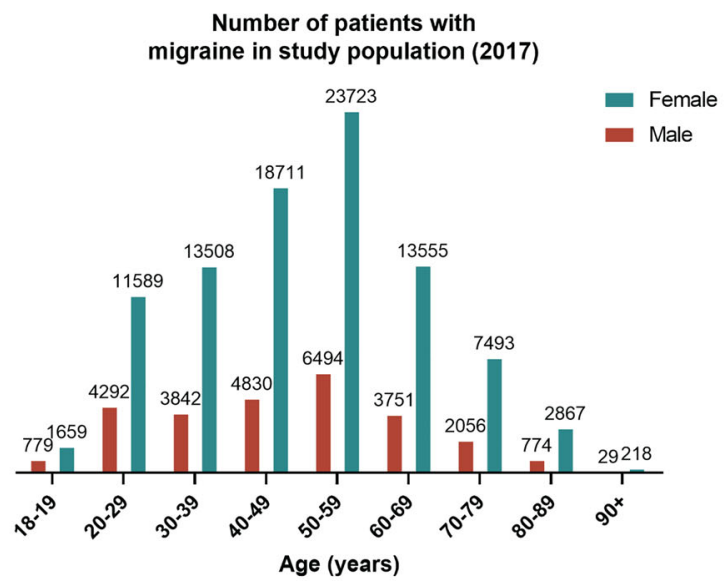

Fig. 1 Sample identification and patient demographics. a Flow chart for the identification of the database population. b Sex distribution and absolute numbers of patients with migraine in the database population. c Absolute numbers of male and female patients with migraine b

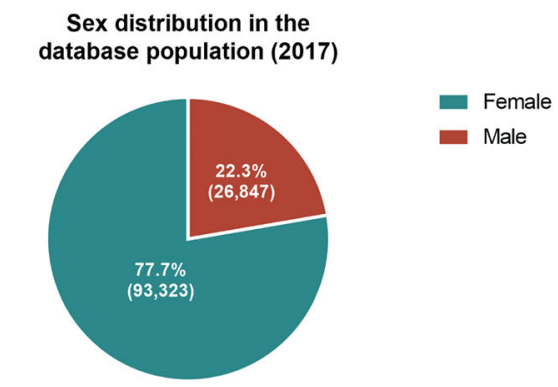

d

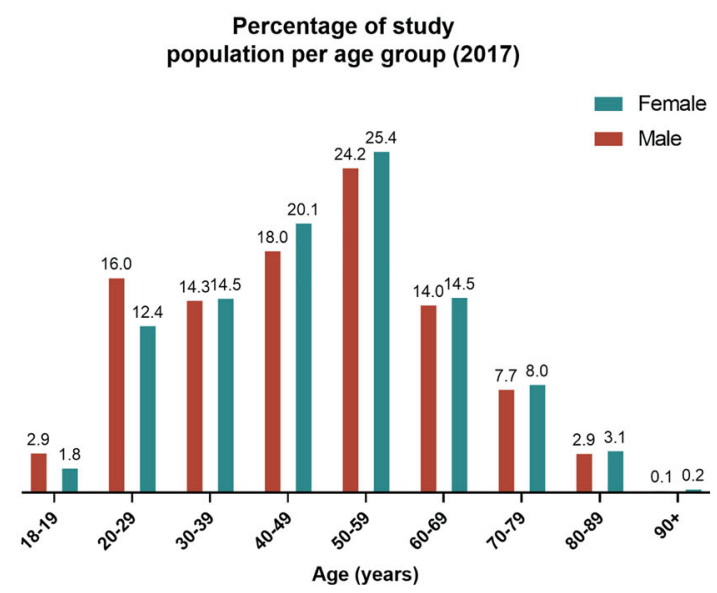

per age category. $\mathbf{d}$ Age distribution of male and female patient with migraine in the database population. ICD-10$G M$ International Classification of Disease 10th Edition; German Modification, $M 2 Q$ criterion at least 2 quarters [“Mindestens zwei Quartale”] 


\section{Data Collection, Analysis and Extrapolation}

Demographic characteristics data (age and sex) was collected for the index year 2017. In the analysis of migraine medication use, medications which were also indicated for diseases other than migraine were only counted if a migraine diagnosis was found within the same quarter. The exception to this rule was triptans since: (1) triptans are only indicated for migraine and cluster headache: (2) cluster headache prevalence is low-reported to be $0.1 \%$-and therefore we can reasonably disregard the possibility of cluster headaches impacting the findings [12, 13]; and (3) all patients were required to have a migraine diagnosis to be included in the database population/analysis. To increase the relevance of findings to the broader German migraine population we extrapolated results from the database to the entire German SHI population. Specifically, data from our 'database population' underwent age- and sex-adjusted extrapolation to the entire German SHI population. In the case of extrapolation, data for the index year 2017 were extrapolated to the year 2019, which at the time of the analysis was the year with the most up-to-date official statistics from the German SHI. The age groups from the German SHI statistics delivered the age groups in 5-year ranges $(15-19,20-24,25-29, \ldots, 90+)$. For an exact extrapolation we used the same 5-years age groups in the claims data analysis. However, based on our inclusion criteria, the formation of the age group 15-19 years was not applicable and therefore we used only the age group 18-19 years. Data extrapolation was done as follows: mean values by sex and age categories were calculated and scaled to the German SHI using statistics from the German Ministry of Health. To approximate the age category of 18-19 years (a 2-year range) from our claims data, the category 15-19 years (a 5-year range) from the SHI statistics was scaled by the factor $2 / 5$. To avoid over-crowding in the figures but still present informative age categories, we summarized the 5-year age groups to 10-year age groups according to the natural age decades. Due to the inclusion of only adult patients, the age group 18-19 could not be included in a 10 -year age group. Consequently, age was categorized into nine groups $(18-19,20-29,30-39$, $40-49,50-59,60-69,70-79,80-89$ and $90+$ years). All analyses were descriptive and performed with appropriate statistical methods using SAS version 9.4 (SAS Institute, Cary, NC, USA). Categorical variables were presented as number and percentage of patients; continuous variables were summarized as the mean and standard deviation (SD).

\section{RESULTS}

\section{Sample Identification and Patient Demographics}

The database population included 120,170 patients with confirmed migraine who were continuously insured between 2010 and 2018 and aged $\geq 18$ years in 2017 (Fig. 1a). Most patients in the database population were female $(77.7 \%, 93,323)$, which is consistent with reported sex-related differences in migraine prevalence (Fig. 1b) [14, 15]. While the overall number of male and female patients with migraine differed, male and female patients in the database population were similarly distributed across all nine age groups analysed; for example, $24.2 \%$ of the male migraine population and $25.4 \%$ of the female migraine population were aged between 50 and 59 years (Fig. 1d). The use of emergency, acute and prophylactic migraine medication by the database population was also documented (2017): 55.4\% of patients in the database population were prescribed acute medications (including triptans) and $24.7 \%$ had received prophylactic medications (Table 1). Overall, $18.2 \%$ of patients in the database populations had received triptan prescriptions (Table 1); of these 21,885 patients prescribed triptans in 2017, $6487(29.6 \%)$ were also being prescribed conventional prophylactics (as described in Table 1) in the same year (data not shown). Finally, $71.2 \%$ of patients with migraine received their first triptan prescription through a general practitioners (GP) office, with $24.4 \%$ receiving their first prescription in consultation with a 
Table 1 Use of acute, prophylactic and emergency medication by all migraine patients in 2017

\begin{tabular}{|c|c|c|c|}
\hline Acute, prophylactic and emergency medication & Prescriptions (mean) & Patients $(n)$ & Patients (\%) \\
\hline$\underline{\text { Acute medication }}$ & $\underline{3.73}$ & $\underline{66,538}$ & $\underline{55.37}$ \\
\hline Propulsives & 1.41 & 6598 & 5.49 \\
\hline Metoclopramide & 1.33 & 5896 & 4.91 \\
\hline Domperidone & 1.81 & 812 & 0.68 \\
\hline Triptans & 3.25 & 21,885 & 18.21 \\
\hline Sumatriptan & 3.10 & 13,044 & 10.85 \\
\hline Oral & 3.07 & 12,624 & 10.51 \\
\hline Nasal & 1.76 & 384 & 0.32 \\
\hline Subcutaneous & 3.46 & 284 & 0.24 \\
\hline Rectal & 1.00 & 1 & 0.00 \\
\hline Eleptriptan & 3.87 & 165 & 0.14 \\
\hline Rizatriptan & 2.67 & 5984 & 4.98 \\
\hline Zolmitriptan & 3.19 & 2801 & 2.33 \\
\hline Oral & 3.04 & 1831 & 1.52 \\
\hline Nasal & 3.09 & 1091 & 0.91 \\
\hline Almotriptan & 2.23 & 81 & 0.07 \\
\hline Naratriptan & 2.93 & 1262 & 1.05 \\
\hline Frovatriptan & 2.74 & 425 & 0.35 \\
\hline NSAIDs, analgesics and others & 3.06 & 54,693 & 45.51 \\
\hline Naproxen & 1.56 & 2070 & 1.72 \\
\hline Acetylsalicylic acid & 2.46 & 2909 & 2.42 \\
\hline Ibuprofen & 1.68 & 29,523 & 24.57 \\
\hline Metamizole & 2.02 & 22,519 & 18.74 \\
\hline Diclofenac & 1.58 & 8665 & 7.21 \\
\hline Paracetamol & 2.77 & 855 & 0.71 \\
\hline Acetylsalicylic acid (combinations) & 1.69 & 278 & 0.23 \\
\hline Ketoprofen & 1.47 & 34 & 0.03 \\
\hline Dexketoprofen & 1.55 & 279 & 0.23 \\
\hline Opioids & 3.59 & 10,249 & 8.53 \\
\hline Ergotamine & 2.57 & 47 & 0.04 \\
\hline Coxibe & 1.72 & 3496 & 2.91 \\
\hline Other analgesics & 1.85 & 1160 & 0.97 \\
\hline Prophylactic medication & $\underline{3.51}$ & $\underline{29,732}$ & 24.74 \\
\hline
\end{tabular}


Table 1 continued

\begin{tabular}{llll}
\hline Acute, prophylactic and emergency medication & Prescriptions (mean) & Patients $(\boldsymbol{n})$ & Patients (\%) \\
\hline Propranolol & 3.67 & 934 & 0.78 \\
Metoprolol & 3.25 & 10,386 & 8.64 \\
Bisoprolol & 2.92 & 8666 & 7.21 \\
Flunarizine & 2.11 & 356 & 0.30 \\
Valproate & 3.51 & 441 & 0.37 \\
Topiramate & 2.93 & 1648 & 1.37 \\
Amitriptyline & 2.48 & 4256 & 3.54 \\
Onabotulinum toxin A & 2.96 & 329 & 0.27 \\
Opipramol & 2.41 & 2941 & 2.45 \\
Magnesium compounds & 7.49 & 74 & 0.06 \\
Lisinopril & 3.09 & 850 & 0.71 \\
Candesartan & 3.73 & 4479 & 0.06 \\
Emergency medication & $\underline{1.65}$ & $\underline{1673}$ & $\underline{1.39}$ \\
Metoclopramide (IV) & 2.26 & 27 & 0.02 \\
Metamizole (IV) & 1.49 & 84 & 0.07 \\
Acetylsalicylic acid (IV) & 1.19 & 43 & 0.04 \\
Prednisone & 1.59 & 615 & 0.51 \\
Dexamethasone & 1.59 & 976 & 0.81 \\
\hline IV & & &
\end{tabular}

$I V$ Intravenous route

neurologist (data not shown). This trend remained the same for all subsequent prescriptions analysed.

\section{Extrapolated Migraine Prevalence by Age and Sex in the German SHI}

We considered a patient as prevalent in 2017 if the $\mathrm{M} 2 \mathrm{Q}$ criterion (described above) was met between 2010 and 2018, and if $\geq 1$ migraine diagnosis was made in 2017. Migraine prevalence was observed to be identical for both our database population and the extrapolated German SHI population, with a migraine prevalence of $2.0 \%$ in males and $7.3 \%$ in females (Fig. 2a). The extrapolation process resulted in 584,463 male and 2,339,516 female patients with migraine in the entire German SHI (2019), with the highest numbers observed in the 50- to 59-year age category (Fig. 2b).

\section{Triptan Contraindications in the German Migraine Population}

The extrapolation process led to a total of $2,923,979$ patients with migraine in the entire German SHI population (2019), of whom 425,089 (14.5\%) had $\geq 1$ triptan contraindication (Fig. 3a). The most frequent triptan contraindication was a history of stroke or transient ischaemic attack; moderate-to-severe/uncontrolled or untreated mild hypertension was ranked second, and coronary/ischaemic heart disease was ranked third (Fig. 3b). Prevalence of 
a

\begin{tabular}{|c|c|c|c|}
\hline \multicolumn{4}{|c|}{ Migraine prevalence extrapolated to German SHI } \\
\hline & Male & Female & Total \\
\hline Rate (\%) & 2.0 & 7.3 & 4.8 \\
\hline $\begin{array}{c}\text { Number of } \\
\text { migraine patients }\end{array}$ & 584,463 & $2,339,516$ & $2,923,979$ \\
\hline
\end{tabular}

b

Extrapolated number of patients with migraine (2019)

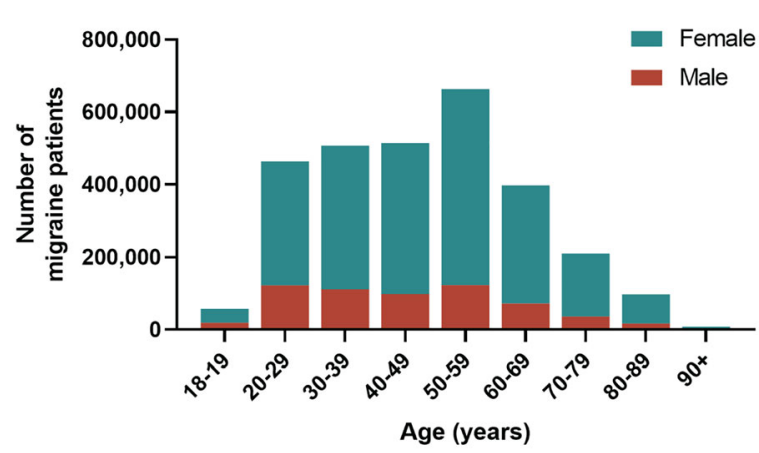

Fig. 2 Migraine prevalence by age and sex extrapolated to the entire German SHI population. a Prevalence and number of patients with migraine in the German SHI population. b Number of patients with migraine by sex and age category in the German SHI population. SHI German Statutory Health Insurance scheme

triptan contraindications increased with age, likely reflecting age-related changes in overall health, with individuals more likely to acquire triptan contraindications over time (Fig. 3c). Absolute numbers of patients in the entire German SHI population contraindicated for triptan use is shown in Fig. 3d. With respect to total number of triptan contraindications observed in the database population, $71.5 \%$ of patients with triptan contraindications had one contraindication, $19.9 \%$ had two, $6.3 \%$ had three and $2.3 \%$ had between four and six different triptan contraindications (data not shown).

\section{Use of Triptans by Patients with Contraindications}

We calculated that an extrapolated 110,265 $(25.9 \%)$ of all patients with migraine and the presence of a triptan contraindication received a triptan prescription following diagnosis of that contraindication (Fig. 4a). A higher percentage of female than male patients per age group have had triptans prescribed despite the presence of a triptan contraindication (Fig. 4b). In addition, we observed that a greater proportion of younger patients with contraindications were prescribed triptans (Fig. 4b). While patient age is not a formal contraindication present in the triptan SmPC, it is a factor taken into consideration by physicians during the therapeutic decision-making process. With respect to the older patient population who did not have any formal triptan contraindication and were actively using triptans in 2019 , only $6.0 \%$ were aged $>64$ years (data not shown).

\section{Overview of Triptan Prescription Patterns and Description of the Population Discontinuing Triptan}

We estimated that 975,698 patients in the entire German SHI population without triptan contraindications had $\geq 1$ triptan prescription within the extrapolated index year of 2019 or during the previous 7 years. Of that population, an extrapolated 596,364 individuals (61.1\%) did not receive a triptan prescription in the followup year. From this subgroup, 576,122 (96.6\%) received a migraine diagnosis after their last triptan prescription (Fig. 5a). This results in 59\% of the analysed 975,698 patients with migraine who had already discontinued their triptan before or during 2019 (Fig. 5a). We termed this population 'triptan discontinuers', $82.6 \%$ of whom were female and $17.4 \%$ male, which reflects the relative sex distribution of patients with migraine in the database population (Fig. 5b). The prevalence of triptan discontinuation was distributed evenly across age and sex groups, with only minor differences observed (Fig. 5c). In the database population, from all patients we defined as triptan discontinuers, $24.0 \%$ had still used triptans in 2017 but would go on to discontinue thereafter, and $76.0 \%$ had already discontinued their triptan use prior to 2017 (see observed data in Table 2). In a further analysis of patients who received a migraine diagnosis after discontinuing their triptan use, 
a

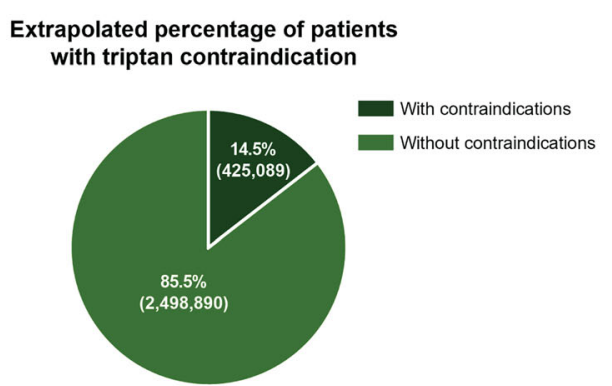

C

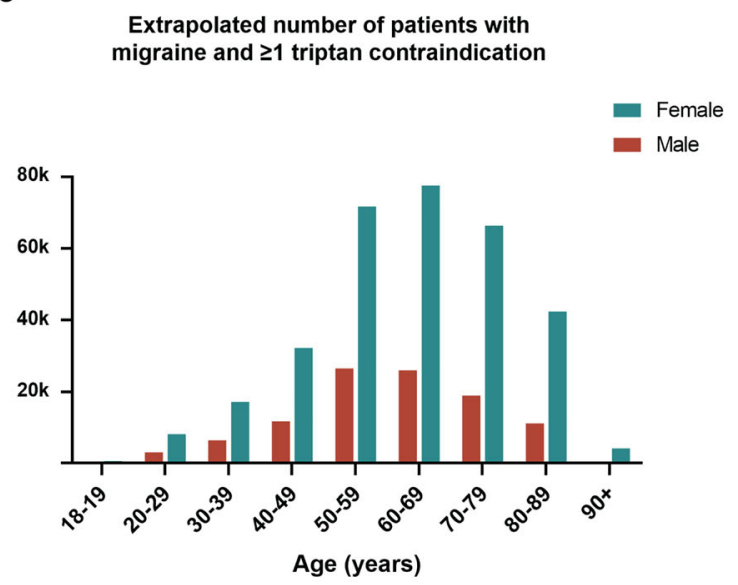

Fig. 3 Triptan contraindications in the entire German SHI migraine population. a Percentage of patients with migraine with $\geq 1$ triptan contraindication, extrapolated to the entire German SHI population. b List of the 7 most frequent triptan contraindications in the entire German SHI population. For hypertension only hypertensive crisis, malignant essential hypertension and hypertensive heart/ renal disease were considered. c Number of patients with

we observed that $68.6 \%$ of these patients were prescribed NSAIDs following cessation of triptans (Table 3).

\section{Triptan Prescription Patterns}

We explored triptan use patterns in our database population, specifically looking at the number of different triptans used and the number of different prescriptions received by patients using a single triptan. We focused our analysis on three patient populations: (1) those still using triptans in 2018 ('ongoing triptan users'); (2) those who discontinued triptans and had no migraine diagnosis thereafter; and (3) b

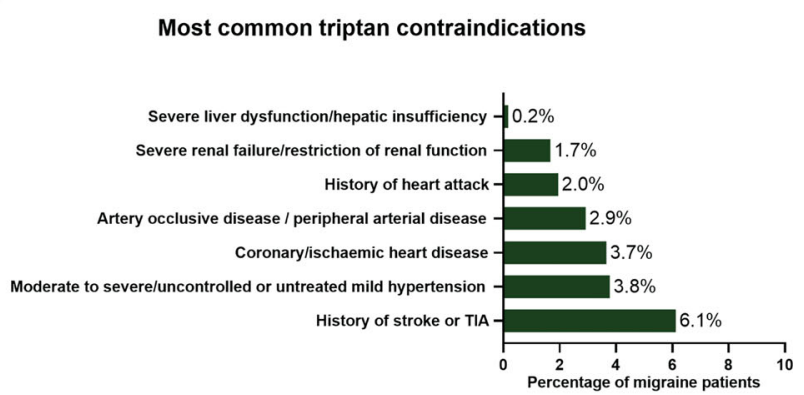

d

Extrapolated prevalence of patients with migraine and $\geq 1$ triptan contraindication

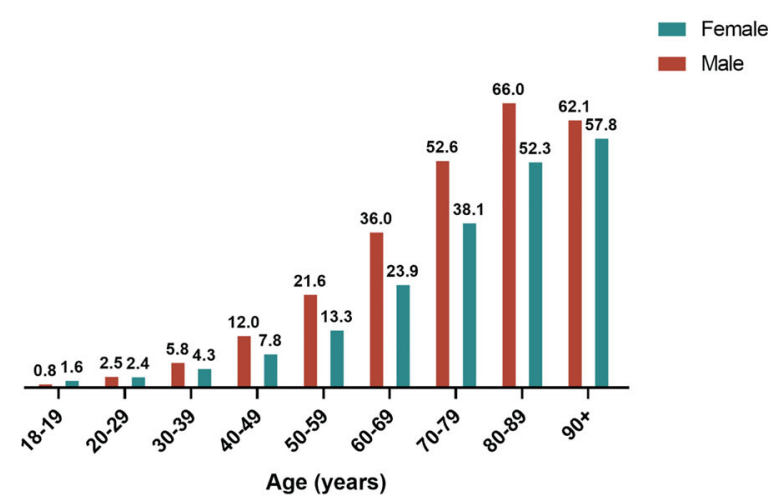

migraine with $\geq 1$ tripan contraindication by sex and age categories in the entire German SHI population. d Prevalence of $\geq 1$ tripan contraindication in patients with migraine by sex and age categories in the entire German SHI population. SHI Statutory Health Insurance, TIA Transient Ischaemic Attack

those who received a migraine diagnosis following triptan discontinuation ('triptan discontinuer' population). We found that most patients, regardless of triptan use, only utilized a single triptan, with little intergroup variability observed (Fig. 6a). Only the group who were still receiving triptans in the follow-up year used a variety of different triptans in higher proportions compared to the other two groups (Fig. 6a). In the triptan discontinuer group, the numbers of different triptans used could have been affected by differences in the timespan between the start of the observation period (2010) and the year of their last triptan prescription (any time between 2010 and 2017). To 
a

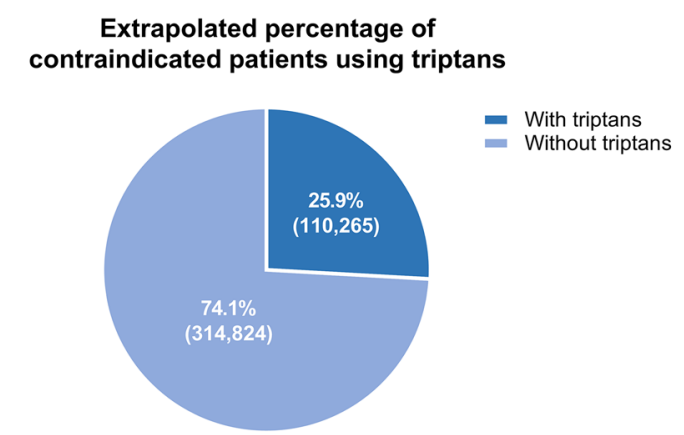

b

Extrapolated prevalence of triptan use by patients with $\geq 1$ triptan contraindication

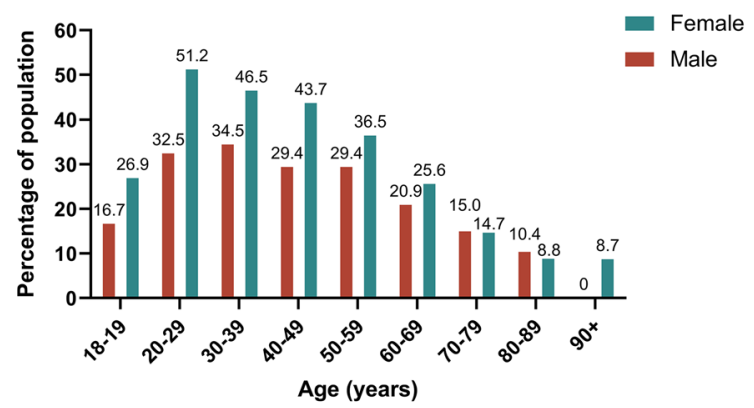

Fig. 4 Use of triptans by patients with contraindications in the entire German SHI migraine population. a Extrapolated percentage and number of patients with migraine in the entire German SHI using triptans despite the presence of $\geq 1$ triptan contraindication. b Prevalence of triptan prescriptions in patients with a minimum $\geq 1$ triptan contraindication by age and sex category, extrapolated to the entire German SHI population

address this possibility, we further considered only those patients who received their last triptan in 2017 (recent triptan discontinuers). We obtained comparable results, with $74 \%$ for one triptan, $21 \%$ for two triptans and $4 \%$ for three triptans; the remaining patients had between four and six different triptans prescribed (data not shown). These findings are consistent with the results of triptan use patterns in patients who had discontinued triptans in the years spanning 2010 and 2017 (Fig. 6a). A large majority of ongoing triptan users who had used a single triptan had received $>4$ prescriptions for that triptan (Fig. 6b). In contrast, most patients who had used a single triptan in the past, but who had discontinued their triptan use, had only one prescription for that triptan (Fig. 6b).

\section{DISCUSSION}

Our analysis used administrative claims data to describe the demographic characteristics and triptan prescription patterns of German patients with migraine. We calculated that within a 1-year period, 1,001,211 patients with migraine in the entire German SHI population either had triptan contraindications, had already discontinued triptans or would discontinue their triptan use after their current prescription. In total, this represents $34.2 \%$ of all patients with migraine in the entire German SHI population. We calculated that over one quarter of patients with migraine and triptan contraindications continued to be prescribed triptans even if they were diagnosed with a triptan contraindication. Additionally, we found that a small proportion of patients received a strikingly high number of triptan prescriptions, and that most German patients with migraine received their first triptan prescription from their GP rather than from a neurologist.

In this study we reported an extrapolated migraine prevalence of $4.8 \%$ (2019), which is lower than previously published prevalence data obtained from population-based epidemiological studies, which vary between $10.6 \%$ (2004) and 14\% (2010-2012) [4, 16-18]. However, the migraine prevalence we found is in line with recently published calculations derived from German administrative claims data that demonstrated an overall administrative prevalence of $4.0 \%$ (2016) [19]. This difference in reported migraine prevalence between claims- and population-based studies may be influenced by several factors. It is worth noting that our analyses only captured those patients with migraine who interacted with the health system of the German SHI; consequently, they may not be reflective of the entire German migraine population. We know that a large proportion of people with migraine may not visit a physician/seek professional care for their condition and in consequence are not documented in the SHI statistics; for example, those 

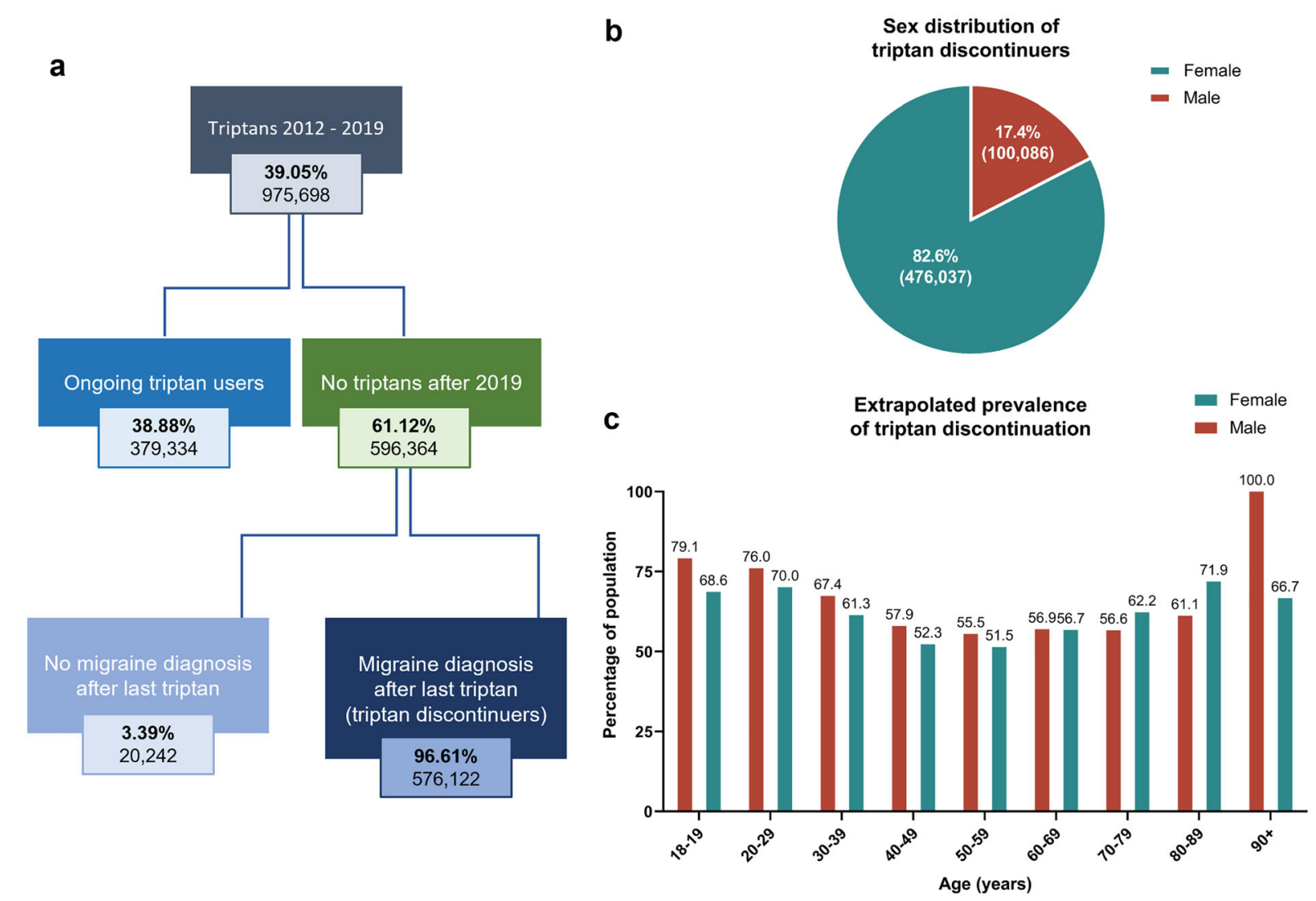

Fig. 5 Overview of triptan use patterns and analysis of triptan discontinuation by patients with migraine without triptan contraindications in the entire German SHI population. a Flow chart depicting the use of triptans by German patients with migraine and identification of an extrapolated 576,122 patients who received a migraine diagnosis after discontinuing use of triptans (termed

who are less severely affected or are satisfied with the effect of over-the-counter medications. Furthermore, Eurolight, a cross-sectional, questionnaire-based survey, revealed a consistent underdiagnosis and undertreatment of migraine across ten European countries, with only approximately one half of the patients who could benefit from consulting a physician doing so [20].

In 2012, a nationally representative study of the German population carried out through computer-assisted telephone interviews found that both self-awareness (patients) and medical recognition (physicians) of migraine was low [21]. Therefore, we may have biased our analysis towards those individuals who feel the need/see 'triptan discontinuers'). b Extrapolated numbers and sex distribution of the triptan discontinuer population. $\mathbf{c}$ Extrapolated prevalence of triptan discontinuation within the total triptan experienced patient population categorized by age and sex

utility in consulting a physician regarding their migraine attacks. With respect to sex differences in migraine, we report a male to female ratio of $1: 4$ in our extrapolated population with migraine. This is consistent with sex ratios of between 1:2 and 1:6 reported for populationbased studies, and identical to ratios (1:4) calculated from similar claims database analyses $[19,22]$.

One major finding from this study is that over one quarter of all patients with migraine who have been diagnosed with a triptan contraindication go on to receive a triptan prescription. While the available data do not allow for further interpretation of this finding, possible reasons could include: (1) the physician is 
Table 2 Year of last triptan prescription for triptan discontinuers

\begin{tabular}{lc}
\hline $\begin{array}{l}\text { Triptan discontinuers; year of last triptan } \\
\text { prescription }\end{array}$ & $\begin{array}{l}\text { Patients } \\
(\%)\end{array}$ \\
\hline 2010 & 7.0 \\
2011 & 7.9 \\
2012 & 8.3 \\
2013 & 9.5 \\
2014 & 11.8 \\
2015 & 13.7 \\
2016 & 17.8 \\
2017 & 24.0 \\
\hline
\end{tabular}

unaware of the contraindication; (2) the physician rates the risk associated with the contraindications as minor; or (3) the physician does not have a viable alternative therapy available for use. Indeed, several studies have shown the cerebrovascular risk associated with triptans in daily practice is very low [23]. We calculated that a higher percentage of younger male and female patients with migraine with $\geq 1$ contraindication were being prescribed triptans. This finding may reflect the physician's belief that younger patients with contraindications have an overall lower risk for serious side effects due to triptan use, with physicians being more confident in prescribing triptans to younger age groups than older age groups. Overall, in the population of patients with migraine without triptan contraindications, we calculated that only $6.0 \%$ of patients aged $>64$ years were being prescribed triptans.

Here we report that in 2019, an extrapolated $59.0 \%$ of patients in the entire German SHI population who have ever used triptans were triptan discontinuers. This percentage of patients who discontinue triptans is slightly higher than that reported in previous studies [24-28]. One possible explanation for this is our consideration of all patients who have used triptans at any point within the 8-year

Table 3 Use of non-steroidal anti-inflammatory drug, analgesics and other medications by triptan discontinuers after last triptan prescription extrapolated to the entire German Statutory Health Insurance population

\begin{tabular}{lcc}
\hline Use of NSAIDs, analgesics, and others after last prescribed triptan & Yes (\%) & No (\%) \\
\hline & 68.6 & 31.4 \\
\hline $\begin{array}{l}\text { Prevalence of using NSAIDs, } \\
\text { analgesics and other medications by } \\
\text { triptan discontinuers after last triptan by age group (years) }\end{array}$ & Male (\%) & \\
\hline $18-19$ & & 51.6 \\
$20-29$ & 44.4 & 59.4 \\
$30-39$ & 53.8 & 65.5 \\
$40-49$ & 64.1 & 69.9 \\
$50-59$ & 70.7 & 76.7 \\
$60-69$ & 75.9 & 79.1 \\
$70-79$ & 78.7 & 84.0 \\
$80-89$ & 78.8 & 83.3 \\
$90+$ & 90.9 & 100.0 \\
\hline
\end{tabular}

NSAID non-steroidal anti-inflammatory drug 
a

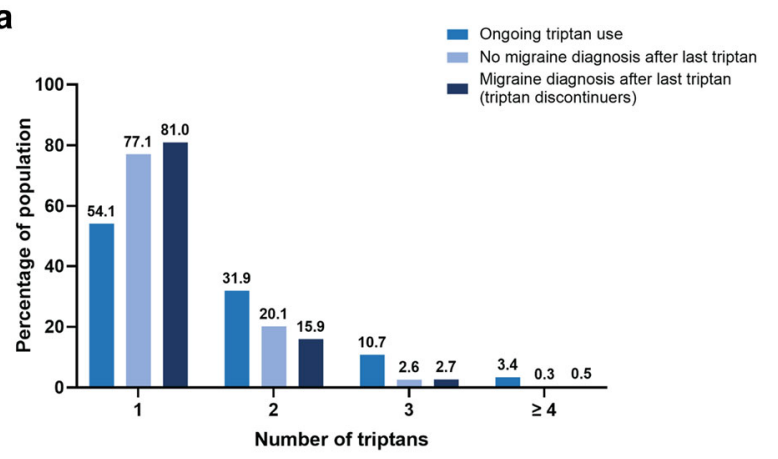

b

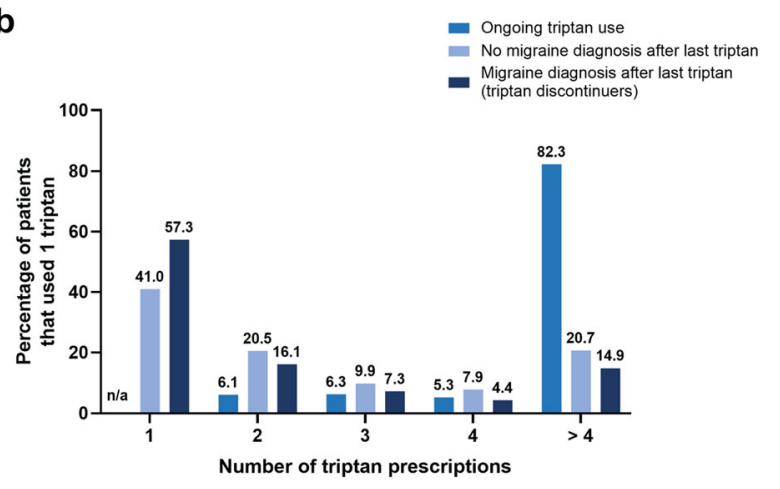

Fig. 6 Triptan prescription and use patterns in the database population. a Observed number of different triptans used by patients with migraine in the database population. b Observed percentage of patients with migraine (that used a single triptan) who received 1, 2, 3,4 , or $>4$ prescriptions for that single triptan

observation period. This approach captured patients who have discontinued triptans in the 7 years prior to the index year in addition to 'new' triptan discontinuers who will stop using triptans within the 1-year follow-up period. In contrast, previous studies have primarily documented the triptan discontinuation rate among 'new' triptan users or discontinuation within a 1-year follow-up period [24-28]. Our analysis identifies new triptan discontinuers and patients who discontinued triptan several years previously but continued to consult their physician. Although the reasons for discontinuation are not documented in the SHI system, it is possible that both these patient populations require alternative therapeutic options. Another interesting observation derived from these data is that $68.6 \%$ of patients with migraine who discontinued their triptan use continued to manage their migraine condition with NSAIDs, analgesics and other medications. While the reasons for this is unclear, it can be speculated that triptans did not provide adequate relief to these patients or they experienced unwanted side effects, with the result that these patients returned to managing their migraine with other readily available non-migraine-specific medications. Further, many people with migraine remain formally undiagnosed, but manage their condition using self-medication with over-thecounter drugs and/or complimentary medicine; data on these patients are not captured in our study.

During the timeframe of our study, both naratriptan and almotriptan were available as over-the-counter triptan treatment options for German patients with migraine [29]. This combined with the preference of certain patients with migraine to manage their condition with NSAIDs and easily accessible medications, such as acetylsalicylic acid and paracetamol, may result in an underestimation of migraine prevalence in the claims database and a skewing of the database population to represent patients that may have more frequent, more severe and more difficult to treat migraine.

In our database population we observed patients with migraine who discontinued triptans after having used only a single triptan prior to discontinuation. This is despite clinical recommendations which advise that in the case of triptan failure, patients with migraine could be switched to another triptan, as failure of a single triptan does not preclude efficacy of another $[30,31]$. With respect to our analyses of the number of triptan prescriptions per patient, information on the size of the package per prescription, which can vary from 2 to 18 dosages (e.g. tablets) per prescription, was not available. Thus, our findings do not allow for the estimation of the number of patients with migraine who are at risk of overusing triptans or analgesics. In the context of the treatment of migraine the International Headache Society classification considers drug overuse to be either the regular use of triptan on $>10$ days per month or the use of analgesics on $>15$ days per month [32]. The proportion of patients with migraine in our database population receiving $>4$ triptan prescriptions may belong to this 
group. We also observed that most German patients with migraine received their first triptan prescription through a GP as opposed to a neurologist. This observation is likely a reflection of the fact that triptans have been in clinical use for $>30$ years and that GPs are comfortable with the relative safety profile of these compounds. Finally, we documented the use of opioids in our database population and observed that $8.5 \%(10,249$ patients) used opioids in 2017. This percentage is surprisingly high given that opioids are not recommended in the guidelines for treating migraine [32-37]. It cannot be ruled out that patients used opioids to treat their comorbidities rather than their migraine (e.g. for men and women, unspecific back pain was the most common comorbidity; for men, other intervertebral disc disorders and spondylosis also appeared in the 10 most common comorbidities [data not shown]).

A limitation of our study is that data on overthe-counter medication, detailed clinical data (e.g. disease activity, graded severity of disease, symptom scores, clinical test results, quality of life data and documentation of prescribed doses, i.e. 'days of supply') and the reason for discontinuation of prescribed triptans are unavailable. It cannot be ruled out that patients with migraine switched from prescribed triptans to over-the-counter triptans or did not have the need for triptans any more due to the start of an effective preventive medication treatment during this time, or that for some patients the follow-up period was too short to capture possible refills of the triptan packages. In addition, we noted that in certain instances, the contraindications as described in the triptan SmPC did not fit exact disease codes as per the ICD-10-GM. To avoid an overestimation of the number of patients with contraindications, we selected only those contraindications with definite ICD10-GM codes. Following the completion of our analysis in 2018, the migraine treatment landscape has evolved, with the emergence of a new class of monoclonal antibodies targeting the calcitonin gene-related peptide (CGRP) and its receptor [38]. These subcutaneous injection therapies have been approved for use as preventive treatments in Germany since late 2018 and may alter the treatment and triptan use patterns in the migraine population. Finally, due to the inclusion criteria (continuously insured 2010-2018 and age $\geq 18$ years in 2017) applied in this study there is a greater contribution of older age groups to the study population, resulting in a slight underestimation of the younger age groups as compared to previous studies [39]. However, this is not likely to have a major impact on the results as most data is extrapolated to the entire German SHI population.

\section{CONCLUSION}

Our analysis used administrative claims data to describe the demographic characteristics and triptan prescription patterns of patients with migraine in Germany. We confirmed the existence of a population of German patients who discontinue triptans despite subsequent migraine diagnoses. We also described the prevalence of triptan contraindications and the concomitant use of triptans by individuals diagnosed with a triptan contraindication. While we cannot determine the exact reasons for triptan discontinuation from our analyses, these results confirm the existence of a population of German patients with migraine who may have unmet therapeutic needs. More research is needed to ascertain reasons for triptan discontinuation and the relative risk of triptan use in patient populations with diagnosed triptan contraindications.

\section{ACKNOWLEDGEMENTS}

Funding. Sponsorship for this study and payment of the journal's Rapid Service Fee was provided by Eli Lilly and Company.

Medical Writing, Editorial, and Other Assistance. Writing support and editorial assistance was provided by Dr. Luke M. Healy, an employee of Eli Lilly and Company, and editorial support was provided by Dana Schamberger, MA, of Syneos Health (Eli Lilly and Company funded this assistance). The 
authors also wish to acknowledge the contributions of Michael Friedrichs (Team Gesundheit GmbH, Essen, Germany) for his statistical analysis and reviewing of the manuscript.

Authorship. All named authors meet the International Committee of Medical Journal Editors (ICMJE) criteria for authorship for this article, take responsibility for the integrity of the work, and have given their approval for this version to be published.

Authors' Contributions. Nadine Rauer, Susanne Kraemer and Inka Schwerdtner contributed to the study conception, design, and method development. Nadine Rauer contributed to data analysis. All authors were involved in the interpretation and presentation of the data. All authors contributed to the drafting and critical revision of the manuscript.

Disclosures. Nadine Rauer, Susanne Kraemer and Inka Schwerdtner are employees of, and have shares in Eli Lilly and Company. Astrid Gendolla has received personal fees and grant/research funding from Grünenthal, Mundipharma, Abbvie/Allergan, Teva, Amgen, Novartis, Bayer, Hormosan, Perfood, Stada, Lundbeck and Eli Lilly and Company. Andreas Straube reports personal fees from Allergan, Bayer, Sanofi, Eli Lilly and Company, Teva Pharmaceuticals, and Novartis; and grants from the German Research Council and the LudwigMaximilian University.

Compliance with Ethics Guidelines. Access to the regulated data was requested and obtained from the BKK. Anonymised data from the electronic databases of the collaborating SHI funds was gathered in accordance with an approved data privacy concept. The use of the fully anonymized secondary data for health services research was compliant with German federal law; therefore, institutional review board/ethical approval was not required $[40,41]$.

Data Availability. All data generated or analysed during this study are available through the corresponding author.
Open Access. This article is licensed under a Creative Commons Attribution-NonCommercial 4.0 International License, which permits any non-commercial use, sharing, adaptation, distribution and reproduction in any medium or format, as long as you give appropriate credit to the original author(s) and the source, provide a link to the Creative Commons licence, and indicate if changes were made. The images or other third party material in this article are included in the article's Creative Commons licence, unless indicated otherwise in a credit line to the material. If material is not included in the article's Creative Commons licence and your intended use is not permitted by statutory regulation or exceeds the permitted use, you will need to obtain permission directly from the copyright holder. To view a copy of this licence, visit http://creativecommons.org/licenses/by$\mathrm{nc} / 4.0 /$.

\section{REFERENCES}

1. Dodick DW. Migraine. Lancet. 2018;391(10127): 1315-30.

2. GBDH 2016 Headache Collaborators. Global, regional, and national burden of migraine and tension-type headache, 1990-2016: a systematic analysis for the Global Burden of Disease Study 2016. Lancet Neurol. 2018;17(11):954-76.

3. GBD 2017 Disease and Injury Incidence and Prevalence Collaborators. Global, regional, and national incidence, prevalence, and years lived with disability for 354 diseases and injuries for 195 countries and territories, 1990-2017: a systematic analysis for the Global Burden of Disease Study 2017. Lancet. 2018;392(10159):1789-858.

4. Woldeamanuel YW, Cowan RP. Migraine affects 1 in 10 people worldwide featuring recent rise: a systematic review and meta-analysis of communitybased studies involving 6 million participants. J Neurol Sci. 2017;372:307-15.

5. Steiner TJ, Stovner LJ, Jensen R, Uluduz D, Katsarava Z. Lifting the burden: the global campaign against $\mathrm{H}$. Migraine remains second among the world's causes of disability, and first among young women: findings from GBD2019. J Headache Pain. 2020;21(1):137.

6. Steiner TJ, Stovner LJ, Vos T, Jensen R, Katsarava Z. Migraine is first cause of disability in under 50s: will 
health politicians now take notice? J Headache Pain. 2018;19(1):17.

7. GBD 2019 Diseases and Injuries Collaborators. Global burden of 369 diseases and injuries in 204 countries and territories, 1990-2019: a systematic analysis for the Global Burden of Disease Study 2019. Lancet. 2020;396(10258):1204-22.

8. Humphrey PP. The discovery and development of the triptans, a major therapeutic breakthrough. Headache. 2008;48(5):685-7.

9. Lambru G, Andreou AP, Guglielmetti M, Martelletti P. Emerging drugs for migraine treatment: an update. Expert Opin Emerg Drugs. 2018;23(4): 301-18.

10. Kreis K, Neubauer S, Klora M, Lange A, Zeidler J. Status and perspectives of claims data analyses in Germany-a systematic review. Health Policy. 2016;120(2):213-26.

11. Lipton RB, Silberstein SD. Episodic and chronic migraine headache: breaking down barriers to optimal treatment and prevention. Headache. 2015;55(Suppl 2):103-22 (Quiz 123-6).

12. Fischera M, Marziniak M, Gralow I, Evers S. The incidence and prevalence of cluster headache: a meta-analysis of population-based studies. Cephalalgia. 2008;28(6):614-8.

13. Katsarava Z, Obermann M, Yoon MS, et al. Prevalence of cluster headache in a population-based sample in Germany. Cephalalgia. 2007;27(9): 1014-9.

14. Brusa P, Allais G, Scarinzi C, et al. Self-medication for migraine: a nationwide cross-sectional study in Italy. PLoS ONE. 2019;14(1):e0211191.

15. Allais G, Chiarle G, Sinigaglia S, Airola G, Schiapparelli $\mathrm{P}$, Benedetto C. Gender-related differences in migraine. Neurol Sci. 2020;41(Suppl 2):429-36.

16. Radtke A, Neuhauser H. Prevalence and burden of headache and migraine in Germany. Headache. 2009;49(1):79-89.

17. Yoon MS, Katsarava Z, Obermann M, et al. Prevalence of primary headaches in Germany: results of the German Headache Consortium Study. J Headache Pain. 2012;13(3):215-23.

18. Schramm SH, Moebus S, Lehmann N, et al. The association between stress and headache: a longitudinal population-based study. Cephalalgia. 2015;35(10):853-63.

19. Roessler T, Zschocke J, Roehrig A, Friedrichs M, Friedel H, Katsarava Z. Administrative prevalence and incidence, characteristics and prescription patterns of patients with migraine in Germany: a retrospective claims data analysis. J Headache Pain. 2020;21(1):85.

20. Katsarava Z, Mania M, Lampl C, Herberhold J, Steiner TJ. Poor medical care for people with migraine in Europe-evidence from the Eurolight study. J Headache Pain. 2018;19(1):10.

21. Radtke A, Neuhauser H. Low rate of self-awareness and medical recognition of migraine in Germany. Cephalalgia. 2012;32(14):1023-30.

22. Obermann M, Katsarava Z. Epidemiology of unilateral headaches. Expert Rev Neurother. 2008;8(9): 1313-20.

23. Ghanshani S, Chen C, Lin B, Duan L, Shen YA, Lee MS. Risk of acute myocardial infarction, heart failure, and death in patients with migraine treated with triptans. Headache. 2020;60(10):2166-75.

24. Fischer M, Frank F, Wille G, Klien S, Lackner P, Broessner G. Triptans for acute migraine headache: current experience with triptan use and prescription habits in a tertiary care headache outpatient clinic: an observational study. Headache. 2016;56(6):952-60.

25. Panconesi A, Pavone E, Franchini M, et al. Triptans: low utilization and high turnover in the general population. Cephalalgia. 2010;30(5):576-81.

26. Holland S, Fanning KM, Serrano D, Buse DC, Reed ML, Lipton RB. Rates and reasons for discontinuation of triptans and opioids in episodic migraine: results from the American Migraine Prevalence and Prevention (AMPP) study. J Neurol Sci. 2013;326(1-2):10-7.

27. Ng-Mak DS, Chen YT, Ho TW, Stanford B, Roset M. Results of a 2-year retrospective cohort study of newly prescribed triptan users in European nationwide practice databases. Cephalalgia. 2012;32(12): 875-87.

28. Lipton RB, Marcus SC, Shewale AR, Dodick DW, Viswanathan HN, Doshi JA. Acute treatment patterns in patients with migraine newly initiating a triptan. Cephalalgia. 2020;40(5):437-47.

29. Tfelt-Hansen P, Steiner TJ. Over-the-counter triptans for migraine: what are the implications? CNS Drugs. 2007;21(11):877-83.

30. Viana M, Genazzani AA, Terrazzino S, Nappi G, Goadsby PJ. Triptan nonresponders: do they exist and who are they? Cephalalgia. 2013;33(11):891-6.

31. Sacco S, Braschinsky M, Ducros A, et al. European headache federation consensus on the definition of 
resistant and refractory migraine: developed with the endorsement of the European Migraine \& Headache Alliance (EMHA). J Headache Pain. 2020;21(1):76.

32. Headache Classification Committee of the International Headache Society (IHS). The international classification of headache disorders, 3rd edition. Cephalalgia. 2018;38(1):1-211.

33. Evers S, Afra J, Frese A, et al. EFNS guideline on the drug treatment of migraine-revised report of an EFNS task force. Eur J Neurol. 2009;16(9):968-81.

34. Diener HC, Antonaci F, Braschinsky $\mathrm{M}$, et al. European Academy of Neurology guideline on the management of medication-overuse headache. Eur J Neurol. 2020;27(7):1102-16.

35. Ford JH, Jackson J, Milligan G, Cotton S, Ahl J, Aurora SK. A real-world analysis of migraine: a cross-sectional study of disease burden and treatment patterns. Headache. 2017;57(10):1532-44.

36. Bigal ME, Lipton RB. Excessive opioid use and the development of chronic migraine. Pain. 2009;142(3):179-82.

37. Diener HC, Gaul C, Kropp P. Therapie der migräneattacke und prophylaxe der migräne. Nervenheilkunde. 2018;37(10):689-715.
38. Tepper SJ. History and review of anti-calcitonin gene-related peptide (CGRP) therapies: from translational research to treatment. Headache. 2018;58(Suppl 3):238-75.

39. Lipton RB, Bigal ME, Diamond M, et al. Migraine prevalence, disease burden, and the need for preventive therapy. Neurology. 2007;68(5):343-9.

40. Bundesinstitut für Medizinprodukte (BfArM), PaulEhrlich-Institut (PEI). Gemeinsame Empfehlungen des Bundesinstituts für Arzneimittel und Medizinprodukte und des Paul-Ehrlich-Instituts zu Anwendungsbeobachtungen nach $\begin{array}{lllll}\S & \text { Absatz } & 6\end{array}$ Arzneimittelgesetz und zur Anzeige von nichtinterventionellen Unbedenklichkeitsprüfungen nach § 63f Arzneimittelgesetz. 2019. https://www.pei.de/ SharedDocs/Downloads/DE/regulation/klinischepruefung/awb-nis-pass-empfehlungen.pdf?_blob= publicationFile\&v=2. Accessed 10 Nov 2021.

41. Swart E, Gothe H, Geyer S, et al. Good practice of secondary data analysis (GPS): guidelines and recommendations, third revision 2012/2014. Gesundheitswesen. 2015;77(02):120-6. 